\title{
Ultrasound based techniques and transient elastography may not be precise methods for the detection of esophageal varices in liver cirrhosis
}

\author{
Reza Jalli', Mohammad Kazem Hosseini-Asl², Ahad Eshraghian², Bahar Rezaei ${ }^{1}$
}

${ }^{1}$ Department of Radiology, Shiraz University of Medical Sciences, Shiraz, Iran, ${ }^{2}$ Gastroenterohepatology Research Center, Department of Internal Medicine.

\section{To the Editor,}

In an interesting systematic review published in Medical Ultrasound, Bintintan et al concluded that some ultrasound parameters including diameter of the spleen $>15 \mathrm{~cm}$, congestion index of the portal vein $>0.154$ $\mathrm{cm} \times$ sec, portal hypertensive index $>2.08$, liver stiffness $>43.97 \mathrm{kPa}$, portal vein diameter $>13 \mathrm{~mm}$, renal artery resistance index $\geq 0.7$ are useful markers of esophageal varices [1]. The study is well written but some points should be considered. Using non- invasive methods for the prediction of esophageal varices has been a challenging issue in recent decades. Ultrasound based techniques are among non invasive method that has been evaluated for the detection of esophageal varices. Different parameters in sonography such as hepatic venous waveform, spleen bipolar diameter, portal vein diameter, measurements of spleen and liver stiffness have been investigated with conflicting results.

In addition to ultrasonography, fibroscan (transient elastography) has been also utilized as a non invasive method for detection of esophageal varices in different studies with conflicting results. Results of some studies showed that liver stiffness and spleen stiffness as measured by fibroscan is a predictor of esophageal varices in patients with liver cirrhosis [2]. However, a recently published meta-analysis showed that current methods

Received 30.10.2013 Accepted 10.11.2013

Med Ultrason

2014, Vol. 16, No 1, 78-80

Corresponding author: Ahad Eshraghian, MD,

Gatsronterohepatology Research Center, |

Namazi Hospital, Shiraz, Iran

PO Box: 71345-1744, Shiraz, Iran

Phone: \#98-711-6125600

Fax: \#98-711-6276212

E-mail:Eshraghiana@yahoo.com for measuring spleen stiffness have limited accuracy for diagnosis of esophageal varices [3]. In the light of the results of this meta-analysis, routine measurement of liver and spleen stiffness for diagnosis of esophageal varices should be applied conservatively. Furthermore, using transient elastography for measuring liver and spleen stiffness has several drawbacks especially among obese patients, those with narrow intercostals spaces and patients with ascites [4]. It is also highly dependent on the examiners' skills and experiences and thus, should have high reproducibility with excellent intra-observer agreement for using in clinical setting. Other new noninvasive method which has been reported to be more precise than transient elastography for measuring liver and spleen stiffness is acoustic radiation force impulse (ARFI) elastography [5]. Results of a meta-analysis showed that AFRI have higher rate of reliable measurement of liver stiffness than transient elastography and is a good method for assessing liver fibrosis [6]. Some have suggested using both methods for more specific prediction of fibrosis [7].

In a cross-sectional study we have investigated 54 cirrhotic patients by endoscopy, among them 28 patients had esophageal varices. These 28 patients were evaluated by trans-abdominal ultrasonography for varices. An esophageal wall thickness $\geq 5 \mathrm{~mm}$ was considered to be diagnostic for esophageal varices. Twenty four patients had positive ultrasonographic findings. Among these patients, 16 individuals had esophageal varices (true positive) while 8 patients had no varices by endoscopy (false positive). Sensitivity and specifity of trans-abdominal ultrasonography was $69.6 \%$ and $74.2 \%$ respectively. Table I showed grade of esophageal varices detected by endoscopy and ultrasound. Doppler ultrasound was applied to detect hepatofugal venous flow signal within esophageal wall. Hepatofugal venous flow is flow di- 
Table I. Sensitivity of sonography in the detection and different grades of esophageal varices

\begin{tabular}{cccc}
\hline Grade & Endoscopy & Sonography & Sensitivity \\
\hline I & 9 & 4 & $44.4 \%$ \\
II & 7 & 4 & $57.1 \%$ \\
III & 5 & 3 & $60 \%$ \\
IV & 7 & 5 & $71.4 \%$ \\
\hline
\end{tabular}

rected away from liver in portal vein, its intrahepatic and extrahepatic branches and is a marker for esophageal varices. From 54 cirrhotic patients, hepatofugal venous flow was identified in 7 patients. In all of these patients the esophageal wall thickness was above $5 \mathrm{~mm}$ and had esophageal varices confirmed by endoscopy. Although specificity of hepatofugal venous blood for the detection of esophageal varices was $100 \%$, its sensitivity was very low $(13 \%)$.

As a conclusion, using non invasive methods such as ultrasonography and fibroscan may not always be applicable for the prediction of esophageal varices in clinical setting.

\section{References}

1. Binţinţan A, Chira RI, Mircea PA. Non-invasive ultrasoundbased diagnosis and staging of esophageal varices in liver cirrhosis. A systematic review of the literature published in the third millenium. Med Ultrason 2013; 15:116-124.

2. Sharma P, Kirnake V, Tyagi $P$, et al. Spleen stiffness in patients with cirrhosis in predicting esophageal varices. Am J Gastroenterol 2013; 108: 1101-1107.

3. Singh S, Eaton JE, Murad MH, Tanaka H, Iijima H, Talwalkar JA. Accuracy of Spleen Stiffness Measurement in Detection of Esophageal Varices in Patients with Chronic Liver Disease: Systematic Review and Meta-analysis. Clin Gastroenterol Hepatol 2013 Sep 17. doi:pii: S1542-3565(13)01320-7.

4. de Lédinghen V, Vergniol J.Transient elastography (FibroScan). Gastroenterol Clin Biol 2008; 32(6 Suppl 1): 58-67.

5. Rizzo L, Calvaruso V, Cacopardo B, et al. Comparison of transient elastography and acoustic radiation force impulse for non-invasive staging of liver fibrosis in patients with chronic hepatitis C. Am J Gastroenterol 2011;06: 2112-2120.

6. Bota S, Herkner H, Sporea I, et al. Meta-analysis: ARFI elastography versus transient elastography for the evaluation of liver fibrosis. Liver Int 2013; 33: 1138-1147.

7. Sporea I, Şirli R, Popescu A, et al. Is it better to use two elastographic methods for liver fibrosis assessment? World J Gastroenterol 2011; 17: 3824-3829.

\section{Author's response}

\section{Adriana Bințințan}

$1^{\text {st }}$ Medical Clinic, Gastroenterology Department, "Iuliu Hațieganu" University of Medicine and Pharmacy, Cluj-Napoca, Romania

We have appreciated the interest and comments of dr. Ahad Eshraghian regarding our review: "Non-invasive ultrasound-based diagnosis and staging of esophageal varices in liver cirrhosis. A systematic review of the literature published in the third Millennium".

We agree with the remarks of our colleagues that there are discordant results in the literature regarding the utility of grey- and Doppler-ultrasound parameters and Fibroscan measurements as markers of esophageal varices. This discordance was actually repeatedly emphasized within our review.

Some of the ultrasound parameters analyzed in our review were proven in the literature to reach statistical significance at certain cut-off values (diameter of the spleen $>15 \mathrm{~cm}$, congestion index of the portal vein $>$ $0.154 \mathrm{~cm} \times \mathrm{sec}$, portal hypertensive index $>2.08$, liver stiffness $>43.97 \mathrm{kPa}$, portal vein diameter $>13 \mathrm{~mm}$, renal artery resistance index $\geq 0.7)$. We have interpreted these results with caution and concluded that, for the moment, the upper gastrointestinal endoscopy still remains the investigation of choice for diagnosis of esophageal varices and evaluation of their risk of hemorrhage. However, we consider that these non-invasive markers do represent an additional tool which may offer important information about the presence and staging of esophageal varices and, probably their significance 
will increase further when they will be coupled within complex algorithms, a possible starting point for future research in this field.

We also remind our colleagues that, in an attempt to increase the scientific accuracy of our review, we have used strict inclusion and exclusion criteria and therefore the number of papers that were selected for the final analyze was limited. By the time we have finalized our work, we haven't found any paper which compared the results of the Acoustic Radiation Force Impulse (ARFI) technique with the presence and development of esopha- geal varices in patients with liver cirrhosis to fulfill the inclusion criteria. We agree that ARFI, alone or in combination with Fibroscan, may bring important information regarding the progression of portal hypertension and development of esophageal varices but this statement still has to be proven in well designed prospective trials.

In this context we do believe that the original study of our Iranian colleagues, shortly presented in the letter, is interesting and we eagerly wait for the publication of the final results. 